\title{
An active state of the BL Lacertae object Markarian 421 detected by INTEGRAL in April 2013*
}

\author{
E. Pian ${ }^{1,2,3}$, M. Türler ${ }^{4}$, M. Fiocchi ${ }^{5}$, R. Boissay ${ }^{4}$, A. Bazzano ${ }^{5}$, L. Foschini ${ }^{6}$, F. Tavecchio 6 , V. Bianchin ${ }^{1}$, \\ G. Castignani ${ }^{7}$, C. Ferrigno ${ }^{4}$, C. M. Raiteri ${ }^{8}$, M. Villata ${ }^{8}$, V. Beckmann ${ }^{9}$, F. D’Ammando ${ }^{10,11,12}$, R. Hudec ${ }^{13,14}$,

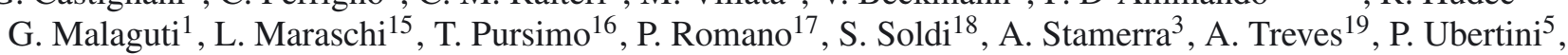 \\ S. Vercellone ${ }^{17}$, and R. Walter ${ }^{4}$
}

1 INAF, Istituto di Astrofisica Spaziale e Fisica Cosmica, via P. Gobetti 101, 40129 Bologna, Italy e-mail: elena.pian@sns.it

2 Scuola Normale Superiore, Piazza dei Cavalieri 7, 56122 Pisa, Italy

3 INFN, Sezione di Pisa, Largo Pontecorvo 3, 56127 Pisa, Italy

${ }^{4}$ ISDC, Geneva Observatory, University of Geneva, Chemin d'Ecogia 16, 1290 Versoix, Switzerland

5 Istituto di Astrofisica e Planetologia Spaziali, via Fosso del Cavaliere 100, 00133 Roma, Italy

6 INAF - Osservatorio Astronomico di Brera, via Bianchi 46, 23207 Merate (LC), Italy

7 SISSA-ISAS, via Bonomea 265, 34136 Trieste, Italy

8 INAF - Osservatorio Astronomico di Torino, Strada Osservatorio 20, 10025 Pino Torinese (TO), Italy

9 François Arago Centre, APC, Université Paris Diderot, CNRS/IN2P3, 10 rue Alice Domon et Léonie Duquet, 75205 Paris Cedex 13, France

10 Dipartimento di Fisica, Università degli Studi di Perugia, via A. Pascoli, 06123 Perugia, Italy

11 INFN, Sezione di Perugia, via A. Pascoli, 06123 Perugia, Italy

12 INAF, Istituto di Radioastronomia, via P. Gobetti 101, 40129 Bologna, Italy

13 Astronomical Institute, Academy of Sciences, Fricova 298, 25165 Ondrejov, Czech Republic

${ }^{14}$ Czech Technical University in Prague, Faculty of Electrical Engineering, 16636 Praha 6, Czech Republic

15 INAF - Osservatorio Astronomico di Brera, via Brera 28, 20100 Milano, Italy

16 Nordic Optical Telescope, Apartado 474, 38700 Santa Cruz de La Palma, Spain

17 INAF, Istituto di Astrofisica Spaziale e Fisica Cosmica, via U. La Malfa 153, 90146 Palermo, Italy

18 APC, Université Paris Diderot, CNRS/IN2P3, 10 rue Alice Domon et Léonie Duquet, 75025 Paris Cedex 13, France

19 Università degli Studi dell'Insubria, via Valleggio 11, 22100 Como, Italy

Received 2 July 2013 / Accepted 9 September 2014

\section{ABSTRACT}

\begin{abstract}
Aims. Multiwavelength variability of blazars offers indirect, but very effective, insight into their powerful engines and on the mechanisms through which energy is propagated from the centre down the jet. The BL Lac object Mkn 421 is a TeV emitter, a bright blazar at all wavelengths, and therefore an excellent target for variability studies.

Methods. We activated INTEGRAL observations of Mkn 421 in an active state on 16-21 April 2013, and complemented them with Fermi-LAT data.

Results. We obtained well sampled optical, soft, and hard X-ray light curves that show the presence of two flares and time-resolved spectra in the 3.5-60 keV (JEM-X and IBIS/ISGRI) and 0.1-100 GeV (Fermi-LAT) ranges. The average flux in the 20-100 keV range is $9.1 \times 10^{-11} \mathrm{erg} \mathrm{s}^{-1} \mathrm{~cm}^{-2}(\sim 4.5 \mathrm{mCrab})$ and the nuclear average apparent magnitude, corrected for Galactic extinction, is $V \simeq 12.2$. In the time-resolved X-ray spectra, which are described by broken power laws and, marginally better, by log-parabolic laws, we see a hardening that correlates with flux increase, as expected in refreshed energy injections in a population of electrons that later cool via synchrotron radiation. The hardness ratios between the JEM-X fluxes in two different bands and between the JEM-X and IBIS/ISGRI fluxes confirm this trend. During the observation, the variability level increases monotonically from the optical to the hard X-rays, while the large LAT errors do not allow a significant assessment of the $\mathrm{MeV}-\mathrm{GeV}$ variability. The cross-correlation analysis during the onset of the most prominent flare suggests a monotonically increasing delay of the lower frequency emission with respect to that at higher frequency, with a maximum time-lag of about $70 \mathrm{~min}$, that is however not well constrained. The spectral energy distributions from the optical to the $\mathrm{TeV}$ domain were compared to homogeneous models of blazar emission based on synchrotron radiation and synchrotron self-Compton scattering. They represent a satisfactory description, except in the state corresponding to the LAT softest spectrum and highest flux.

Conclusions. Multiwavelength variability of Mkn 421 can be very complex, with patterns changing from epoch to epoch down to intra-day timescales, depending on the emission state. This makes accurate monitoring of this source during bright hard X-ray states necessary and calls for the elaboration of multicomponent, multizone, time-dependent models.
\end{abstract}

Key words. galaxies: active - X-rays: galaxies - radiation mechanisms: non-thermal - gamma rays: galaxies

\footnotetext{
* Based on observations obtained with INTEGRAL, an ESA mission with instruments and science data centre funded by ESA member states (especially the PI countries: Denmark, France, Germany, Italy, Switzerland, Spain, Czech Republic, and Poland), and with the participation of Russia and the USA.
} 


\section{Introduction}

Blazars are active multiwavelength extragalactic sources, with compact inner engines powered by supermassive black holes and with relativistic jets pointing at small angles with respect to the observer. They are the most luminous persistent sources in the Universe, reaching bolometric luminosities as large as $10^{49} \mathrm{erg} / \mathrm{s}$, often dominated by the gamma-ray output (Ghisellini et al. 2011), and are highly variable, with doubling timescales ranging from seconds to years. Recent dense and accurate monitorings have shown that, while in general the variation amplitudes are higher at shorter wavelengths (e.g. Pian et al. 2011), the multiwavelength behaviour is often more complex and calls into question the role of multiple radiation components (Aleksić et al. 2012; Bonning et al. 2012; D'Ammando et al. 2013). The blazar spectrum is characterised by a thin synchrotron component that peaks, in a $v f_{v}$ representation, in the wavelength range from far-infrared to X-rays, and by an inverse Compton component that extends from $\mathrm{X}$-rays to the $\mathrm{TeV}$ range, peaking at $\mathrm{MeV}-\mathrm{GeV}$ energies (Falomo et al. 2014). These characteristic frequencies vary in an anti-correlated way with respect to bolometric luminosity (Fossati et al. 1998; see however Padovani et al. 2012), leading to a broad distinction of blazars into low-frequency-peaked and high-frequency-peaked objects (Padovani \& Giommi 1995). Assuming that the emitting region responsible for the emission at X-ray and gamma-ray energies is homogeneous, the ratio between the peak frequencies of the two components represents the characteristic cooling energy of the relativistic particles in the jet plasma (barring Klein-Nishina suppression).

Mkn $421(z=0.031)$ is a known bright BL Lac object (Ulrich 1973; Colla et al. 1975) of the high-frequencypeaked type, a strong and variable X-ray source up to the hard $\mathrm{X}$-rays, and belongs to the complete sample of X-ray blazars selected above $15 \mathrm{keV}$ by the Swift/Burst Alert Telescope survey (Ajello et al. 2009). The absence of strong optical emission lines indicates that photon sources external to the jet, e.g. an accretion disk or a dust torus, are modest and do not contribute significantly to the electron cooling. As a consequence, the synchrotron component peaks at relatively high frequencies also during quiescence (soft-X-rays) and the synchrotron photons are the main targets for Compton up-scattering by the relativistic particles. This synchrotron self-Compton component peaks at very high energies, making this blazar a strong $\mathrm{TeV}$ emitter, although the TeV spectrum is often heavily suppressed by the Klein-Nishina effect (Maraschi et al. 1999; Fossati et al. 2008; Mankuzhiyil et al. 2011). Mkn 421 was the target of many multiwavelength campaigns involving high energy satellites, Cherenkov telescopes and ground-based optical and radio facilities (Takahashi et al. 1996; Maraschi et al. 1999; Malizia et al. 2000; Fossati et al. 2000a,b; Brinkmann et al. 2001; Albert et al. 2007a; Lichti et al. 2008; Fossati et al. 2008; Donnarumma et al. 2009; Acciari et al. 2009; Ushio et al. 2009; Tramacere et al. 2009; Horan et al. 2009; Aleksić et al. 2010, 2012; Isobe et al. 2010; Abdo et al. 2011; Acciari et al. 2011; Barres de Almeida 2011; Shukla et al. 2012). The variations at $\mathrm{X}$-ray and $\mathrm{TeV}$ frequencies are often very well correlated with no measurable delay (Maraschi et al. 1999), but complex intra-day $\mathrm{X}$-ray variability is present (e.g. Tanihata et al. 2001).

In 2013, Mkn 421 has undergone a prolonged state of high activity, with a peak around January 2013, and revived episodes in April 2013. Many instruments detected this high state: Swift, Fermi, NuSTAR, MAXI, ground-based optical, and
TeV (Baloković et al. 2013; Cortina, \& Holder 2013; Paneque et al. 2013; Negoro et al. 2013; Semkov et al. 2013; Krimm et al. 2013), and radio/mm wavelengths (Hovatta et al. 2013). We activated our programme for INTEGRAL follow-up of blazars in outburst, and started observing on 16 April 2013 with the IBIS/ISGRI, JEM-X, and OMC instruments. The results of this campaign are presented here along with those of the simultaneous Fermi-LAT observations.

\section{Observations and results}

\subsection{INTEGRAL}

Mkn 421 was observed as a Target of Opportunity by INTEGRAL (Winkler et al. 2003) in the periods 2013 April 16.13-18.58 UT (Revolution 1283) and 19.20-21.68 UT (Revolution 1284) for $200 \mathrm{ks}$ each time. The effective net exposure time was $270.9 \mathrm{ks}$ for IBIS/ISGRI (Ubertini et al. 2003; Lebrun et al. 2003) and $345.7 \mathrm{ks}$ for JEM-X (Lund et al. 2003). We observed in hexagonal dithering mode, so that the source was always in the JEM-X field of view. The screening, reduction, and analysis of the INTEGRAL data have been performed using the INTEGRAL Offline Scientific Analysis (OSA) V. 10.0, publicly available through the INTEGRAL Science Data Centre (ISDC, Courvoisier et al. 2003a,b). The algorithms implemented in the software are described in Goldwurm et al. (2003) for IBIS and Westergaard et al. (2003) for JEM-X.

\subsubsection{OMC}

The OMC (Mas-Hesse et al. 2003) data were acquired with a standard $V$-band Johnson filter and extracted with default settings, using a $3 \times 3$ pixels binning, which is appropriate for pointlike sources. Individual measurements that were flagged as problematic were disregarded. The target is well detected. From the OMC data-points we subtracted both the contribution of the host galaxy and that of a companion galaxy located at about 14 arcsec north-east of the nucleus (see Ulrich 1978; Gorham et al. 2000; Nilsson et al. 2007). For the former we adopted $R=13.29 \pm 0.02$ from Hubble Space Telescope imaging and a colour $V-R=0.63$ (Urry et al. 2000). This magnitude was obtained by integrating the galaxy radial profile to infinity, which is appropriate, considering that the adopted OMC photometric aperture corresponds to a diameter of 50 arcsec, which is equivalent to infinity for practical purposes (the host of Mkn 421 has a half-light radius of only a few arc-sec). For the companion galaxy we used the SDSS photometry reported in the NED ${ }^{1}$ database (this source is identified as RXJ1104.4+3812:BEV[98]014), converted to the Johnson $V$ band. Our total estimated flux of this satellite galaxy in the $V$-band is $1.72 \pm 0.02 \mathrm{mJy}$. Finally, we have applied a Galactic extinction correction using $E_{B-V}=0.014$ (Schlafly \& Finkbeiner 2011) and the curve of Cardelli et al. (1989).

The corrected OMC fluxes are reported in Fig. 1a. Two flares, of which only the first is fully resolved, are detected. The first outburst is symmetrical, with rising and decay times (defined as the intervals during which the flux increases or decreases from quiescence to peak, respectively) of 1 day each in the observer frame. Taking the uncertainties into account, the variation amplitude from quiescence to maximum flux is $20 \%$. The second outburst shows an increase of similar amplitude in 1.5 days, while the decay is not fully sampled. Our average flux $(48.5 \pm 0.4 \mathrm{mJy})$ is approximately consistent with the

http://ned.ipac.caltech.edu 


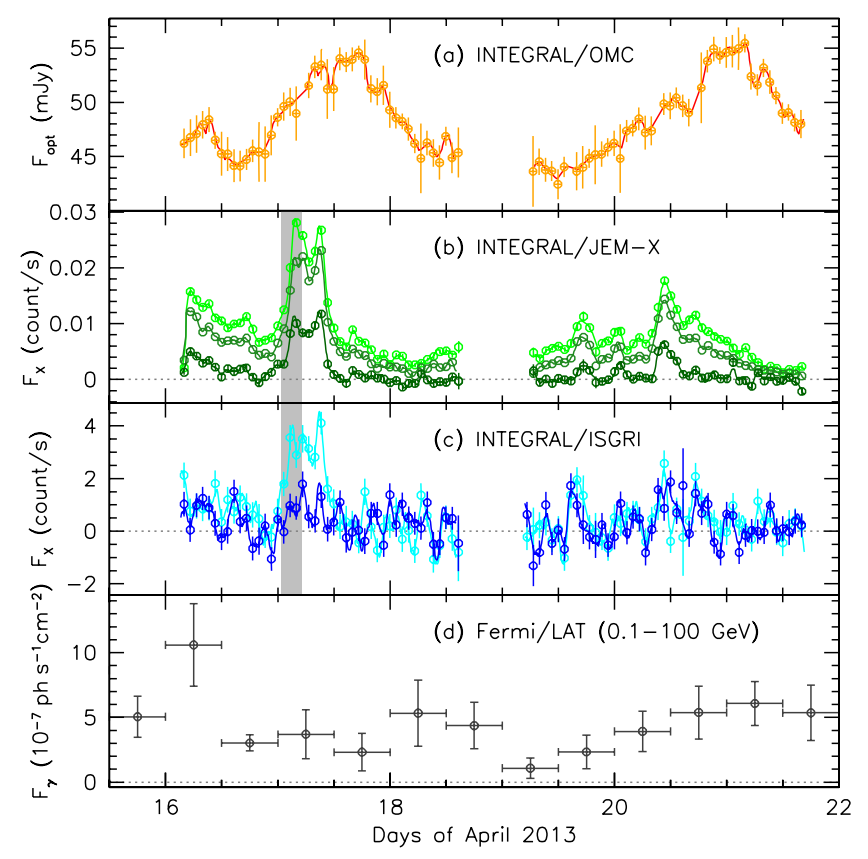

Fig. 1. Light curves of Mkn 421 in April 2013: a) OMC dereddened $\left(E_{B-V}=0.014\right)$ photometry, corrected for the host galaxy and for a companion galaxy that is located in the OMC field of view; b) JEM-X count rates (resulting from the coaddition of the signal measured by the 2 detectors), in the bands 3.04-5.52 keV (light green), 5.52-10.24 keV (green), and 10.24-25.88 keV (dark green); c) IBIS/ISGRI count rates at 20-40 keV (light blue) and 40-100 keV (dark blue); d) Fermi-LAT fluxes binned with $12 \mathrm{~h}$ time-resolution. As INTEGRAL provides quasi-continuous datasets, we display them both as data points with a binning of $4.8 \mathrm{ks}$ and as a curve representing a weighted smoothing of the data (see Sect. 3 for details). The shaded grey area in panels b) and c) represents the interval chosen for the crosscorrelation analysis (see Fig. 5).

photometry obtained just prior to our monitoring (Semkov et al. 2013), and it is about a factor of 2 larger than measured in June 2006 ( $25 \mathrm{mJy}$ ) by Lichti et al. (2008), when it is taken into account that these authors adopt a somewhat dimmer host galaxy contribution, and do not correct for the presence of the host galaxy companion.

\subsubsection{JEM-X and IBIS}

The source is detected with JEM-X in most individual pointings with a significance of $10 \sigma$. The light curve is reported in Fig. $1 \mathrm{~b}$ in three different bands.

IBIS/ISGRI detected the source significantly in individual science windows ( $2000 \mathrm{~s})$ only on some occasions, notably during maximum flux on April 17, when it reached $8 \sigma$ significance in a single science window. The final mosaic yields an average flux in the $20-100 \mathrm{keV}$ range of $1.10 \pm 0.05$ counts s$^{-1}$, corresponding to $(4.4 \pm 0.2) \mathrm{mCrab}$. The IBIS/ISGRI light curves in the distinct ranges $20-40 \mathrm{keV}$ and $40-100 \mathrm{keV}$ are reported in Fig. 1c. No detection was obtained with IBIS/PICsIT.

As in the optical band, the X-ray observations display two main outbursts detected up to $\sim 40 \mathrm{keV}$, with much higher amplitude (a factor of $\sim 4$ in $5-10 \mathrm{keV}$ in the first outburst and a factor of $\sim 3$ in the second outburst with respect to quiescence) and shorter timescale: while the first outburst appears approximately symmetrical with rising and decay times of $\sim 0.5$ days each, in the second outburst the rise time is less than 0.5 days while the decay lasts about 1 day. The $40-100 \mathrm{keV}$ light curve is affected by large statistical errors, so that assessing variability is difficult.

Our JEM-X measurement of the X-ray flux is comparable to that of June 2006 reported by Lichti et al. (2008), while our IBIS/ISGRI measurement is about a factor of 2 lower.

\subsubsection{X-ray spectra}

The overall JEM-X and IBIS/ISGRI spectra were extracted, combined and, after convolution with the most recent matrices available in OSA V. 10.0, fitted in the energy range $3.5-60 \mathrm{keV}$ with a single power law, a broken power law, and with a logparabola of the form $f(E) \propto[E /(10 \mathrm{keV})]^{-(a+b \log [E /(10 \mathrm{keV})])}$ (Massaro et al. 2004a, 2006; Perlman et al. 2005). A fixed intercalibration constant of unity between JEM-X and IBIS/ISGRI was assumed (Jourdain et al. 2008). Photoelectric absorption caused by the neutral hydrogen along this line of sight $\left(N_{\mathrm{H}}=\right.$ $1.43 \times 10^{20} \mathrm{~cm}^{-2}$, Elvis et al. 1989) is negligible at these frequencies, and therefore not corrected for. The fit results are reported in the last row of Table 1.

In order to study spectral variability in different states of the source, we defined a series of time intervals for which we extracted JEM-X $1 \& 2$ spectra and IBIS/ISGRI spectra. We chose to investigate separately the rising and the decaying phases of the two main flares, i.e. the periods April 16.93-17.1 and April 17.42-17.65 for the first flare, and the periods April 20.21-20.48 and April 20.48-20.78 for the second flare. In addition, we selected the time period April 16.13-16.52, which is simultaneous to the phase of highest $\mathrm{GeV}$ emission, the extended period of high flux during the first flare from April 17.1 to 17.42, and a quiescent state obtained by coadding the signal during the periods April 17.77 to 19.58 and April 21.04 to 21.7, to increase the signal-to-noise ratio.

We then fitted the joint JEM-X and IBIS/ISGRI spectra (3.5-60 keV) with a single power law, a broken power law, and a log-parabola model, using the same assumptions for JEM-X vs. IBIS/ISGRI inter-calibration and Galactic absorption as adopted for the average spectrum. The results are reported in Table 1 and in Fig. 2. For the average and all time-resolved joint JEM-X and IBIS/ISGRI spectra the broken power law and the log-parabola yield better fits than the single power law, and the log-parabola is preferable over the broken power law because it is marginally better constrained ( 3 vs. 4 free parameters). While the break energy parameter of the broken power law fits and the $a$ parameter of the log-parabola model both vary significantly (the constancy probability is less than 0.02 ), the $\log$-parabola $b$ parameter only varies marginally (constancy probability of 0.35 ). Accordingly, the correlation of the break energy and $a$ parameter with flux is more significant than that of the $b$ parameter with flux: the weighted linear correlation coefficient between the break energy and the integrated flux is $r=0.73$, with a probability of 0.062 of chance correlation; the weighted linear coefficients between the $\log$-parabola indices $a$ and $b$ and the flux are $r=-0.85$ (probability of 0.015 ) and $r=-0.66$ (probability of 0.11 ), respectively (Bevington \& Robinson 2003).

\subsubsection{Hardness ratios}

As done in Lichti et al. (2008), we have computed the hardness ratios in the energy bands covered by IBIS/ISGRI and JEM-X. Specifically, we have first computed the ratio between the simultaneous fluxes in the $20-40 \mathrm{keV}$ and $40-100 \mathrm{keV}$ ranges. Unlike 


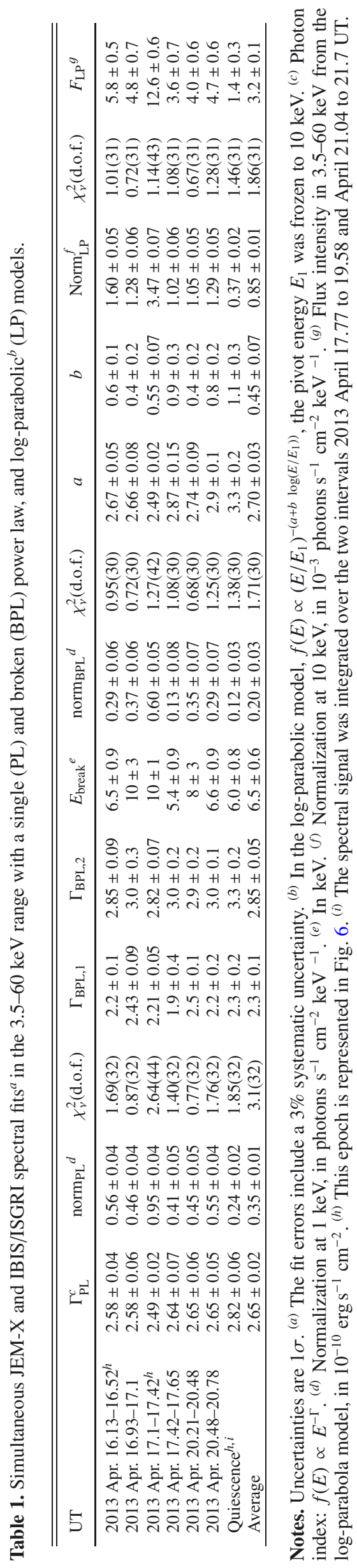

in the IBIS/ISGRI observations of June 2006, when the gammaray flux was higher than presently found (Lichti et al. 2008), this ratio does not vary significantly within the large errors, nor is there any significant correlation of the hardness ratio with either the softer or the harder IBIS/ISGRI band flux. Then we evaluated the ratio between the simultaneous fluxes in the whole IBIS/ISGRI range (20-100 keV) and in the softest JEM-X band (3.04-5.52 keV). The variability behaviour of this ratio traces that of the light curves (Fig. 1), i.e. it indicates spectral hardening accompanying flux brightening. Although the result has a limited significance (see Fig. 3a), it is confirmed by the correlation of hardness ratio with both the gamma- and X-ray flux (Fig. 3c), and it is consistent with the findings of spectral analysis (see Fig. 2). Finally we have computed the ratios of the simultaneous fluxes in the JEM-X ranges $3.04-5.52 \mathrm{keV}$ and $10.24-25.88 \mathrm{keV}$. These follow a behaviour similar to that of the IBIS/ISGRI vs. JEM-X hardness ratios: the ratio between these X-ray bands varies in a similar way as the JEM-X light curves (Fig. 3b) and it increases with flux, both when the $10.24-25.88 \mathrm{keV}$ and the 3.04-5.52 keV fluxes are considered (Fig. 3d). Again, this indicates correlated spectral hardening with source brightening, as often seen in Mkn 421 at these energies. No different dependencies are seen between hardness ratio and flux during brightening vs. dimming phases (hysteresis cycles), as previously reported by Takahashi et al. (1996) and Fossati et al. (2000b) in X-rays.

\subsection{Fermi-LAT}

Data of Mkn 421 covering the period 15-22 April 2013 were downloaded from the LAT online public archive ${ }^{2}$ and analysed with standard methods ${ }^{3}$. In particular, we used a zenith angle of 100 degrees and a rocking angle of 52 degrees. The adopted software is LAT Science Tools v. 9.27.1, with the Instrument Response Function P7SOURCE_V6 and corresponding files for the Galactic diffuse and isotropic background. The extraction region has a radius of interest of 10 degrees and is centred on the radio position of Mkn 421. The sources of the 2FGL Catalog present within this radius and Mkn 421 itself were modelled with single power laws by leaving the parameters free. We have verified that by adopting a somewhat bigger radius, 15 degrees, our results do not change. In order to verify the robustness of the source detection we used the test statistics (TS) method (Mattox et al. 1996), taking a threshold of $T S=25$ (equivalent to about $5 \sigma$ ). The light curve is reported in Fig. 1d.

The LAT state is similar and marginally brighter than detected by Abdo et al. (2011) and a factor of 3 brighter than reported in the 2FGL catalog (Nolan et al. 2012), after reducing all fluxes to the $0.1-100 \mathrm{GeV}$ range. We extracted the LAT spectra over time intervals of 12 and $24 \mathrm{~h}$ and fitted them to single power laws $\left(f(E) \propto E^{-\Gamma}\right)$ in the range $0.1-100 \mathrm{GeV}$. A correlation between flux and photon index is seen in the sense of spectral hardening for flux dimming (Fig. 4), which is similar to previous behaviour seen in Mkn 421 in the period August 2008-March 2010, and in another blazar of the HBL class, PKS 2155-304 (Foschini et al. 2010). We have tested this correlation quantitatively (see e.g. Albert et al. 2007b; Acciari et al. 2009; Aharonian et al. 2009; Anderhub et al. 2009). We performed a test of constancy both on photon indices and fluxes, an error-weighted least squares linear fit and a weighted linear

\footnotetext{
2 http://fermi.gsfc.nasa.gov/cgi-bin/ssc/LAT/ LATDataQuery.cgi

3 http://fermi.gsfc.nasa.gov/ssc/data/analysis/ scitools/
} 
E. Pian et al.: INTEGRAL observations of Mkn 421
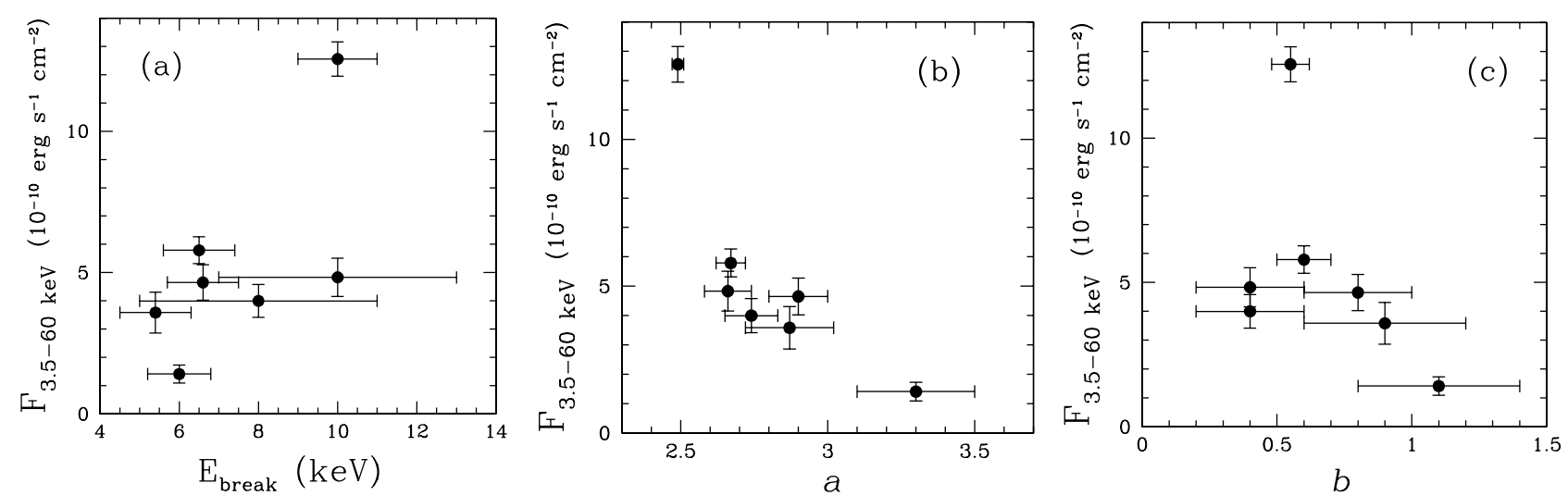

Fig. 2. Flux integrated in the joint JEM-X and IBIS/ISGRI range (3.5-60 keV), as derived from the log-parabola spectral fits, vs. a) break energy, as derived from the broken power law fits of time-resolved spectra; b) $a$ index from log-parabola fits; c) $b$ index from log-parabola fits.
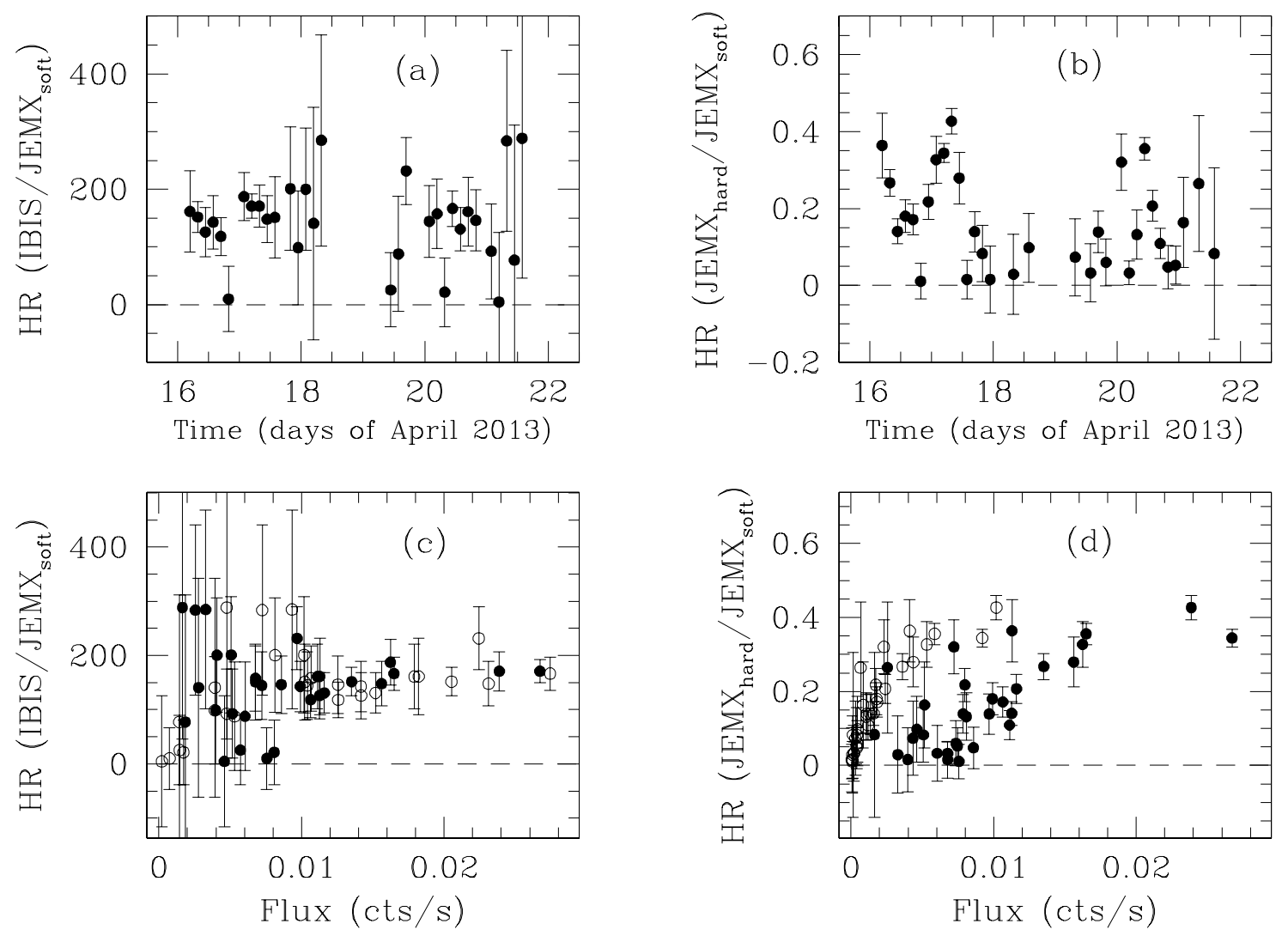

Fig. 3. a) Ratio between IBIS/ISGRI (20-100 keV) flux and JEM-X (3.04-5.52 keV) flux as a function of time; b) ratio between JEM-X 10.24-25.88 keV flux and 3.04-5.52 keV flux as a function of time; c) ratio between 20-100 keV flux vs. 3.04-5.52 keV flux as a function of 3.04-5.52 keV flux (filled circles) and 20-100 keV flux reduced by a factor of 100 (open circles); d) ratio between 10.24-25.88 keV flux and $3.04-5.52 \mathrm{keV}$ flux as a function of 3.04-5.52 keV (filled circles) and 10.24-25.88 keV flux multiplied by 2 (open circles). In all cases, the adopted time binning is $3 \mathrm{~h}$.

Pearson correlation coefficient, and computed the associated probabilities according to Bevington \& Robinson (2003). All results are reported in Table 2 . The $12 \mathrm{~h}$ binned photon index is consistent with a constant behaviour, while the flux binned with the same time resolution varies somewhat more significantly. Both the weighted linear fit and the correlation coefficient suggest however a decent correlation, both when the whole dataset is used and when the point of highest flux and steepest spectrum is ignored (see Fig. 4). This suggests that the photon index and flux, albeit modestly variable, are correlated. When the $24 \mathrm{~h}$ binned fluxes and indices are considered, this conclusion is confirmed. We note that for that same period, Abdo et al. (2011) do not report any correlation between flux and photon index for Mkn 421, likely because it cannot be seen with integration times of one week.

\section{Variability and timing analysis}

Following Lichti et al. (2008), we evaluated the variability of our multiwavelength light curves using a fractional rms variability amplitude as defined in Fossati et al. (2000a) and Vaughan et al. (2003). As in Lichti et al. (2008), we find increasing variability 
Table 2. Statistical parameters of Fermi-LAT fluxes and spectral indices correlation.

\begin{tabular}{lcccccc}
\hline \hline Test & $\chi_{12 \mathrm{~h}}^{2}$ & $v_{12 \mathrm{~h}}$ & $P_{12 \mathrm{~h}}{ }^{a}$ & $\chi_{24 \mathrm{~h}}^{2}$ & $v_{24 \mathrm{~h}}$ & $P_{24 \mathrm{~h}}{ }^{a}$ \\
\hline Constant photon index & $9.93(4.37)^{b}$ & $10(9)$ & $0.50(0.89)$ & 6.16 & 6 & 0.47 \\
Constant flux & $17.3(13.0)$ & $10(9)$ & $0.065(0.18)$ & 21.9 & 6 & $<10^{-4}$ \\
Weighted least squares linear fit & $7.0(7.0)$ & $9(8)$ & $0.68(0.59)$ & 4.50 & 5 & 0.54 \\
Weighted correlation coefficient & $0.77(0.68)$ & $9(8)$ & $0.0055(0.03)$ & 0.89 & 5 & 0.0072 \\
\hline
\end{tabular}

Notes. The sub-index in the $\chi^{2}$ value, degrees of freedom $v$ and probability $P$ indicates the time binning of the data: 12 or $24 \mathrm{~h} .{ }^{(a)}$ This indicates the probability of the null hypothesis for the tests of constancy and linear. trend and of chance correlation for the correlation coefficient. ${ }^{(b)}$ The quantities in parentheses for the $12 \mathrm{~h}$ binning parameters refer to the case where the point of steepest spectrum and highest flux was removed.

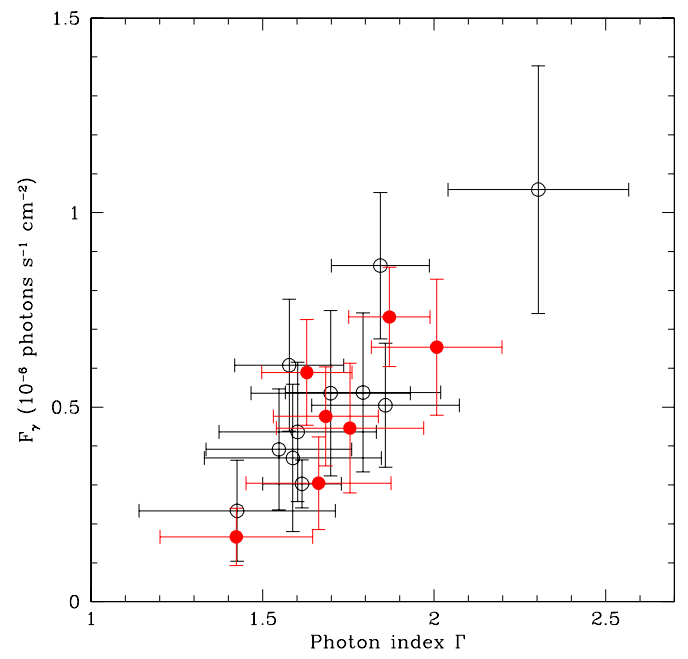

Fig. 4. Fermi-LAT flux $(0.1-100 \mathrm{GeV})$ vs. photon index $\Gamma$ during the campaign. The data are averaged over intervals of $12 \mathrm{~h}$ (black open circles) and $24 \mathrm{~h}$ (red filled circles). Only measurements for which $T S>$ 25 were retained.

Table 3. Fractional rms variability amplitude.

\begin{tabular}{lll}
\hline \hline Instrument & Band & $F_{\text {var }}{ }^{a}$ \\
\hline OMC & $V$-band & $0.065 \pm 0.003$ \\
JEM-X & $3.04-5.52 \mathrm{keV}$ & $0.68 \pm 0.01$ \\
JEM-X & $5.52-10.24 \mathrm{keV}$ & $0.85 \pm 0.01$ \\
JEM-X & $10.24-25.88 \mathrm{keV}$ & $1.78 \pm 0.04$ \\
IBIS/ISGRI & $20-40 \mathrm{keV}$ & $1.4 \pm 0.1$ \\
IBIS/ISGRI & $40-100 \mathrm{keV}$ & $1.5 \pm 0.3$ \\
Fermi-LAT & $0.1-100 \mathrm{GeV}$ & $0.35 \pm 0.14$ \\
\hline
\end{tabular}

Notes. ${ }^{(a)}$ See Fossati et al. (2000a); Vaughan et al. (2003).

at increasing energies, although this trend is reverted at the LAT energies, where the fractional variability is lower than that computed for IBIS/ISGRI (see Table 3), albeit marginally significant. This is partly due to the relatively large errors of the LAT measurements, but it also matches the fact that the $\mathrm{GeV}$ photons are produced by inverse Compton scattering off the electrons that are responsible for the less variable optical spectrum. With respect to Lichti et al. (2008), our variability indices are all larger, indicating larger variability amplitudes in general and a higher level of inter-day and intra-day activity.

We also attempted to cross-correlate the JEM-X and IBIS/ISGRI light curves in search of time lags. We extracted the light curves in various energy ranges in time bins of $300 \mathrm{~s}$ for JEM-X and $600 \mathrm{~s}$ for IBIS/ISGRI and smoothed these oversampled light curves in a time window of $\sim 6 \mathrm{ks}$ with a modified

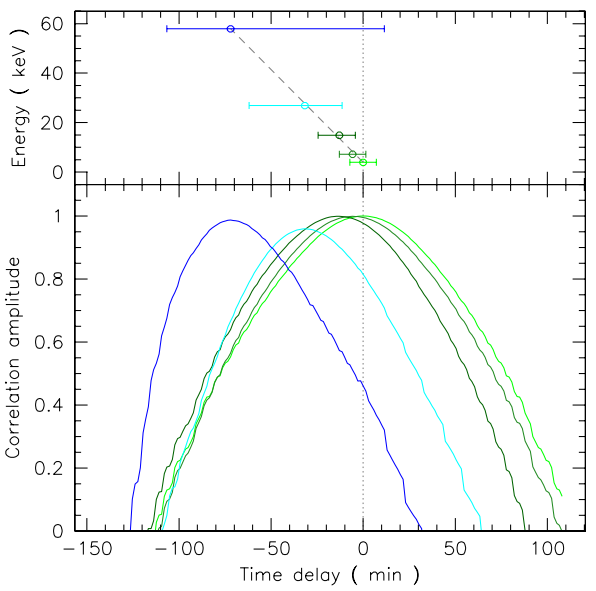

Fig. 5. Cross-correlation function of the INTEGRAL JEM-X (the outputs of the 2 detectors have been coadded) and IBIS/ISGRI light curves during the onset and first peak of the main outburst (April 17.03-17.21 UT). Bottom panel: correlation curves of JEM-X data in the softest range (3.04-5.52 keV) with itself and with the light curves at higher energies, identified by the same colours as in Fig. 1. Top panel: corresponding correlation time lags as a function of energy (same colour-coding as in bottom panel) with $1 \sigma$ uncertainties based on the distribution of lags obtained by perturbing the original light curves (see text). The energy was evaluated by calculating the average photon energy in the spectral band assuming a photon index of $\Gamma=2.8$, as obtained by a single power law fit to the combined JEM-X and IBIS/ISGRI overall spectra. The dashed line in the upper panel simply connects the first and last points to visualise the trend.

boxcar smooth (Türler, in prep.). The averaging inside the time bins is done by taking into account both the measurement errors and sampling times. More weight is given to points with lower uncertainties and closer to the centre of the time window, making the smoothing robust for light curves with an irregular sampling and unequal errors. We used the Interpolated Cross-Correlation Function (ICCF, Gaskell \& Peterson 1987), which is appropriate for curves with no big data gaps, with the improvements introduced by White \& Peterson (1994). The time lag corresponds to the maximum of the correlation curve.

In Fig. 5 (bottom panel) we show the correlation curves of the JEM-X 3.04-5.52 keV light curve with respect to itself (autocorrelation), and with respect to the other bands during the rising phase of the first flare, i.e. when only data in the time window April 17.03-17.21 UT are considered. In the top panel of the figure are reported the corresponding time lags vs. the energy of the band centroid. A negative time lag corresponds to harder photons leading the softer ones. The $1 \sigma$ errors are obtained by calculating the lag on 1000 perturbed light curves and following the flux-randomization/random subset-selection 
method described in Peterson et al. (1998). These perturbed light curves were then smoothed and cross-correlated with the ICCF method. We estimated the $1 \sigma$ statistical uncertainties by taking the boundaries of the region comprising the central $68 \%$ of the distribution of their time lags (i.e. $\pm 34 \%$ from the median). The higher energies light curves lead the softest one by a linearly increasing time lag (a weighted linear least squares fit of the 5 time lags vs. their corresponding energies yields $\chi^{2}=0.118$ with a probability of $99 \%$ ), with a maximum of 72 min between the 40-100 keV and the 3.04-5.52 keV signal. However, this trend is formally not significant because the time lags deviations from a constant lag of -7 min correspond, when error-weighted, to a $\chi^{2}=3.15$ (assuming the upper errors on the time lags, that are on average worse), which yields a constancy probability of $59 \%$ for 4 degrees of freedom.

We explored other time windows around the first and second flares and did not find more compelling evidence for time delays. In particular, the flare of April 20 suggests a similar trend for JEM-X, but the IBIS/ISGRI signal is not significant, whereas the peak and early decay of the April 17 flare is found to be quasi-simultaneous in all X-ray bands. Our time lags are comparable in absolute value to those found for Mkn 421 in the INTEGRAL observations of June 2006 (Lichti et al. 2008), and to those reported by Sembay et al. (2002), Brinkmann et al. (2003), Ravasio et al. (2004) for the X-rays only (see also Zhang et al. 2004). They are also in line with the report of a much larger time lag, 10 days, of the optical vs. X-ray photons (Gaur et al. 2012). These lags can set important constraints on the cooling times of the relativistic particles responsible for the synchrotron radiation, and in turn on the magnetic field.

The two maxima in the OMC light curve might be related to the two main outbursts seen in the X-rays. The overall delay is of $\sim 0.5$ days, as confirmed by a cross-correlation analysis. We did not try the correlation test on the LAT light curve, because of the paucity of flux points.

\section{Discussion}

We observed and detected Mkn 421 with the INTEGRAL instruments IBIS/ISGRI, JEM-X and OMC during a high state that followed the detection of a powerful TeV energy outburst (Cortina \& Holder 2013) that was likely subsiding during our observation. The X-ray flux is among the highest previously recorded for this object by INTEGRAL itself and other satellites (Malizia et al. 2000; Donnarumma et al. 2009; Abdo et al. 2011). The optical state is also very bright, with few precedents (Tosti et al. 1998). While our X-ray flux is similar to that reported by Lichti et al. (2008) for the observations of June 2006, our optical and gamma-ray measurements are a factor of 2 higher and lower, respectively, suggesting that the synchrotron spectrum pivoted around the X-ray frequencies during the 2006 and 2013 states. This also results in a lower break energy than observed by Lichti et al. (2008). This parameter never exceeds $10 \mathrm{keV}$ in our fits, while the X-ray models of Lichti et al. (2008) can accommodate a break energy higher than $\sim 40 \mathrm{keV}$. As suggested by both our models and those presented by Lichti et al. (2008), the peak of the $v f_{v}$ synchrotron spectrum occurs at lower energies $(\sim 1 \mathrm{keV}$ in our multiwavelength models, see Fig. 6).

Besides a broken power law, we have attempted to fit the joint JEM-X and IBIS/ISGRI spectra with a smoothly steepening power law, best described by a log-parabola model, as it is often seen in BL Lacs where the hard X-ray radiation is due to the synchrotron process (Maraschi et al. 1999; Tavecchio et al. 2001; Massaro et al. 2004b; Lichti et al. 2008; Giommi et al. 2012).

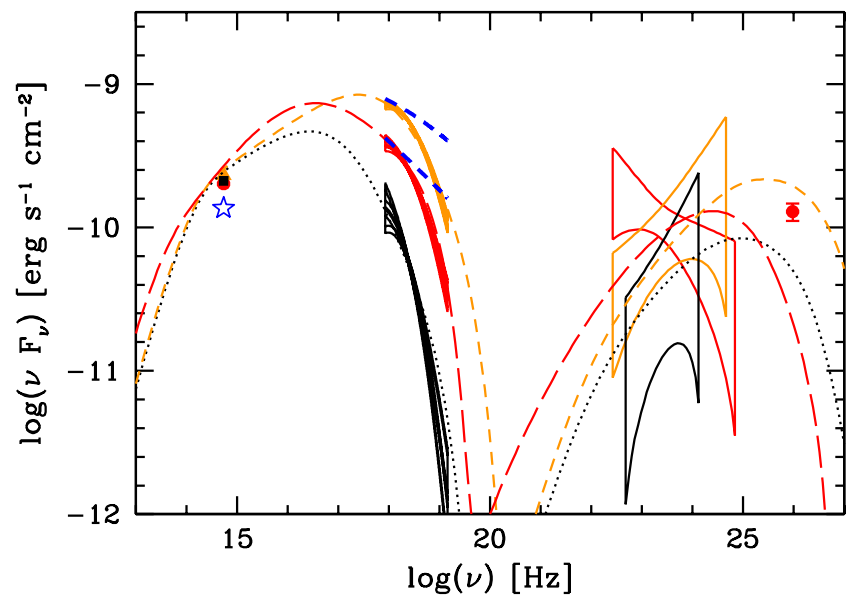

Fig. 6. Spectral energy distributions of Mkn 421 at the average UT epochs of 16.1-16.5 (red circle) and 17.1-17.4 (orange triangle) April 2013 and during the quiescent state (black square), that we relate here to the minimum Fermi-LAT flux of April 19.0-19.5, from simultaneous INTEGRAL IBIS/ISGRI, JEM-X and OMC, and Fermi-LAT data. The optical data were corrected for Galactic absorption and for the contamination by galaxies in the field as described in the text. The $1 \sigma$ error contours of the joint JEM-X and IBIS/ISGRI spectra and LAT spectra are reported. The TeV point (red) was taken on April 16.3 and is the last point of the VERITAS observation (Mukherjee 2013; still preliminary). The models (long dash: April 16, short dash: April 17, dot: April 19) include a synchrotron component at the lower energies, produced in a single emitting zone, and a synchrotron self-Compton scattering component at higher energies (see model parameters in Table 4). For comparison, the JEM-X and IBIS/ISGRI quiescent and active state spectra of June 2006 (Table 7 in Lichti et al. 2008) are reported as thick dashed blue lines. The average optical flux at the same epoch is shown as a blue star.

The X-ray flux computed from the log-parabolic fits correlates decently both with the break energy derived from the broken power law fits, and with the log-parabola $a$ index (Fig. 2). A correlation between flux and break energy and/or spectral indices is expected, because injection of fresh and energetic particles in the emitting region should cause both flux enhancement and spectral hardening. This is seen, with various levels of significance, also in the behaviour of the JEM-X and IBIS/ISGRI hardness ratios (Fig. 3) and in the frequency-dependent variability (Table 3).

We found a weak correlation between LAT flux and photon index, in the sense of a softer spectrum accompanying a higher flux (Fig. 4), which is opposite of what we observe in the INTEGRAL data. This can be explained if the effect is highly dependent on the observing band: when peak energy of the inverse Compton component is located within the LAT range the flux is higher and the spectrum is flat $(\Gamma \sim 2)$, while when it moves to energies higher than the LAT range it causes the flux to decrease and the spectrum to harden $(\Gamma<2)$. Notably, this behaviour seems to be only weakly correlated with the X-ray variations (Fig. 1), and is not observed on timescales longer than $\sim 1$ day (Abdo et al. 2011). Whether systematics or instrumental effects are involved it is difficult to assess, because the phenomenon may trace complex physics, whose signal washes out when mediated on long timescales.

Multiwavelength spectral energy distributions of Mkn 421 from optical to gamma rays were constructed in three representative states (Fig. 6): the first state refers to the initial phase of our INTEGRAL observation (April 16.13-16.52), when LAT measured the brightest flux and softest spectrum; the second state represents the first INTEGRAL flare (April 17.1-17.42), 
Table 4. Model parameters of the multiwavelength energy distributions.

\begin{tabular}{lccc}
\hline \hline Parameter & 2013-Apr.-16 $\left(\right.$ red $\left.^{a}\right)$ & 2013-Apr.-17 (orange) & 2013-Apr.-19 (black) \\
\hline$\gamma_{\min }$ & 1000 & 4000 & 4000 \\
$\gamma_{\mathrm{b}}$ & 31000 & $4.1 \times 10^{5}$ & $1.3 \times 10^{5}$ \\
$\gamma_{\max }$ & $5 \times 10^{5}$ & $2 \times 10^{6}$ & $1 \times 10^{6}$ \\
$n_{1}$ & 2 & 2.5 & 2.5 \\
$n_{2}$ & 3.7 & 4.9 & 4.9 \\
$B(\mathrm{Gauss})$ & 0.6 & 0.038 & 0.04 \\
$K\left(\mathrm{~cm}^{-3}\right)$ & 1800 & $1.9 \times 10^{5}$ & $1.1 \times 10^{5}$ \\
$R(\mathrm{~cm})$ & $1.7 \times 10^{16}$ & $1.3 \times 10^{16}$ & $1.5 \times 10^{16}$ \\
$\delta$ & 10 & 40 & 40 \\
\hline
\end{tabular}

Notes. ${ }^{(a)}$ Colours refer to the coding in Fig. 6.

when the 3.5-60 keV flux was at its brightest and LAT measured an intermediate flux and spectrum; the third state describes the dimmest and hardest LAT measurement of April 19.0-19.5, associated with the quiescent INTEGRAL state (indicated with "quiescence" in Table 1), that we obtained by averaging the flux over two intervals where the $3.5-60 \mathrm{keV}$ flux was lowest. The TeV flux had subsided to quiescence by the time our INTEGRAL observation started, thus we report here the latest flux $(1.75 \pm 0.25 \mathrm{Crab}$ units at $E>400 \mathrm{GeV})$ of the campaign of the VERITAS Cherenkov telescope (Mukherjee 2013), which, recorded on April 16.3 UT, is quasi-simultaneous with the start of our observation. We caution the reader that this measurement is still preliminary. In Fig. 6 are reported for comparison also the observations of June 2006 (Lichti et al. 2008), that show the similarity of the X-ray flux and the difference in optical and gamma-ray state.

We modelled the spectral energy distributions with a singlezone emitting model with a synchrotron component at lower energies, and self-Compton scattering at the higher energies (e.g. Tavecchio et al. 2011). Relevant model parameters include the minimum, break and maximum energy of the electron energy distribution, $\gamma_{\min }, \gamma_{b}, \gamma_{\max }$, respectively; the indices of the distribution below and above the break energy, $n_{1}$ and $n_{2}$, the magnetic field $B$; the particle density in the emitting region $K$, the size of the emitting region or blob $R$, and the Doppler boosting factor $\delta$ (Table 4). The model parameters are in general similar to those determined by Lichti et al. (2008), Donnarumma et al. (2009), and Abdo et al. (2011) in their multiwavelength fits with a leptonic model. Accommodating the softest LAT spectrum and the simultaneous TeV flux proved to be difficult (see Fig. 6). While the discrepancy between the model and the TeV flux may be justified by the very high amplitude variability at these energies on short timescales, the softness of the LAT spectrum is unusual and may command substantial changes in our interpretation of this source. Many authors have previously advocated more complex scenarios than the one we have adopted here, to account for the MeV-GeV spectrum of Mkn 421, such as time-dependent and multicomponent models, lepto-hadronic jets, multizone models, electron energy laws with evolving spectra rather than steady-state spectra, inhomogeneous jets, spineand-layer jet structure (Krawczynski et al. 2001; Błażejowski et al. 2005; Ghisellini et al. 2005; Aharonian et al. 2009; Potter $\&$ Cotter 2013; Mastichiadis et al. 2013; Asano et al. 2014). We believe indeed that a time-dependent approach should primarily lead to a better description of the high-energy spectrum. In view of this, the large variation of the magnetic field inferred from our modelling of the very soft LAT spectrum of April 16 with respect to the two following states may be reduced, when a more complex, but more plausible time-dependent description is implemented.

During the rising part of the first flare of this campaign the softer energy flux seems to lag behind the higher energy flux with a delay that increases linearly with energy, but is formally not significant (Fig. 5). Because of this limitation and because the correlation cannot be tested on other parts of the light curves, we cannot use this to constrain time-dependent models. The complex multiwavelength variability observed in Mkn 421 during the present outburst and previous ones occurred and studied over the past years indicates that the source behaviour may be dominated by different regimes of injection and cooling in different multiwavelength states, and thus must be monitored intensively during the transition phases to high states, in order to reconstruct the physical conditions that produce all regimes of variability.

Acknowledgements. We thank Celia Sanchez, Marion Cadolle-Bel, Erik Kuulkers and Chris Winkler of the INTEGRAL Science Operation Centre for their assistance with the scheduling of the observations, Lucia Pavan for assistance with JEM-X data calibration, Imma Donnarumma for helpful discussion, Eran Ofek for help with computational issues and use of his astronomy \& astrophysics package for Matlab, and the anonymous referee for suggestions and comments that helped to improve the paper. This work was partially supported by ASI/INAF contracts I/009/10/0 and I/088/06/0. R.H. acknowledges GA CR grant 102/09/0997. This research has made use of the NASA/IPAC Extragalactic Database (NED) which is operated by the Jet Propulsion Laboratory, California Institute of Technology, under contract with the National Aeronautics and Space Administration. This paper is dedicated to the memory of our friend and colleague Paul Barr.

\section{References}

Abdo, A. A., Ackermann, M., Ajello, M., et al. 2011, ApJ, 736, 131 Acciari, V. A., Aliu, E., Aune, T., et al. 2009, ApJ, 703, 169 Acciari, V. A., Aliu, E., Arlen, T., et al. 2011, ApJ, 738, 25

Aharonian, F., Akhperjanian, A. G., Anton, G., et al. 2009, ApJ, 696, L150 Ajello, M., Costamante, L., Sambruna, R. M., et al. 2009, ApJ, 699, 603 Albert, J., Aliu, E., Anderhub, H., et al. 2007a, ApJ, 663, 125

Albert, J., Aliu, E., Anderhub, H., et al. 2007b, ApJ, 669, 862

Aleksić, J., Anderhub, H., Antonelli, L. A., et al. 2010, A\&A, 519, A32

Aleksić, J., Alvarez, E. A., Antonelli, L.A., et al. 2012, A\&A, 542, A100 Anderhub, H., Antonelli, L. A., Antoranz, P., et al. 2009, ApJ, 705, 1624 Asano, K., Takahara, F., Kusunose, M., Toma, K., \& Kakuwa, J. 2014, ApJ, 780, 64

Baloković, M., Furniss, A., Madejski, G., \& Harrison, F. 2013, ATel, 4974

Barres de Almeida, U. 2011, Proc. of the 32nd International Cosmic Ray Conference (ICRC2011), held 11-18 August, Beijing, China, OG2.3-2.4: Cosmic Ray Origin and Galactic Phenomena, 8, 78

Bevington, P. R., \& Robinson, D. K. 2003, Data reduction and error analysis for the physical sciences, 3rd edn. (Boston, MA: McGraw-Hill), eds. P. R. Bevington, \& K. D. Robinson

Błażejowski, M., Blaylock, G., Bond, I. H., et al. 2005, ApJ, 630, 130

Bonning, E., Urry, C. M., Bailyn, C., et al. 2012, ApJ, 756, 13 
Brinkmann, W., Sembay, S., Griffiths, R. G., et al. 2001, A\&A, 365, L162 Brinkmann, W., Papadakis, I. E., den Herder, J. W. A., \& Haberl, F. 2003, A\&A, 402, 929

Cardelli, J. A., Clayton, G. C., \& Mathis, J. S. 1989, ApJ, 345, 245

Colla, G., Fanti, C., Fanti, R., et al. 1975, A\&AS, 20, 1

Cortina, J., \& Holder, J. 2013, ATel, 4976

Courvoisier, T. J.-L., Beckmann, V., Bourban, G., et al. 2003a, A\&A, 411, L343

Courvoisier, T. J.-L., Walter, R., Beckmann, V., et al. 2003b, A\&A, 411, L53

D’Ammando, F., Antolini, E., Tosti, G., et al. 2013, MNRAS, 431, 2481

Dickey, J. M., \& Lockman, F. J. 1990, ARA\&A, 28, 215

Diehl, R., Baby, N., Beckmann, V., et al. 2003, A\&A, 411, L117

Donnarumma, I., Vittorini, V., Vercellone, S., et al. 2009, ApJ, 691, L13

Elvis, M., Lockman, F. J., \& Wilkes, B. J. 1989, AJ, 97, 777

Falomo, R., Pian, E., \& Treves, A. 2014, A\&ARv, 22, 73

Foschini, L., Ghisellini, G., Maraschi, L., et al. 2013, Patterns of variability in gamma-ray active galactic nuclei, in Time Variability at High Energies: a Probe of AGN Physics (including VLBI), 38th COSPAR Scientific Assembly, Bremen (Germany), 18-25 July 2010, eds. L. Foschini, G. Tosti, F. Aharonian, et al., ZENODO, DOI: 10.5281/zenodo.7464

Fossati, G., Maraschi, L., Celotti, A., Comastri, A., \& Ghisellini, G. 1998, MNRAS, 299, 433

Fossati, G., Celotti, A., Chiaberge, M., et al. 2000a, ApJ, 541, 153

Fossati, G., Celotti, A., Chiaberge, M., et al. 2000b, ApJ, 541, 166

Fossati, G., Buckley, J. H., Bond, I. H., et al. 2008, ApJ, 677, 906

Gaskell, C. M., \& Peterson, B. M. 1987, ApJS, 65, 1

Gaur, H., Gupta, A. C., \& Wiita, P. J. 2012, AJ, 143, 32

Ghisellini, G., Tavecchio, F., \& Chiaberge, M. 2005, A\&A, 432, 401

Ghisellini, G., Tagliaferri, G., Foschini, L., et al. 2011, MNRAS, 411, 901

Giommi, P., Polenta, G., Lähteenmäki, A., et al. 2012, A\&A, 541, A160

Goldwurm, A., David, P., Foschini, L., et al. 2003, A\&A, 411, L223

Gorham, P. W., Van Zee, L., Unwin, S. C., \& Jacobs, C. 2000, AJ, 119, 1677

Horan, D., Acciari, V. A., Bradbury, S. M., et al. 2009, ApJ, 695, 596

Hovatta, T., Baloković, M., Richards, J. L., Max-Moerbeck, W., \& Readhead, A. C. S. 2013, Atel, 5107

Isobe, N., Sugimori, K., Kawai, N., et al. 2010, PASJ, 62, L55

Jourdain, E., Götz, D., Westergaard, N. J., Natalucci, L., \& Roques, J. P. 2008, PoS (Integral 08) 144

Kalberla, P. M. W., Burton, W. B., Hartmann, D., et al. 2005, A\&A, 440, 775

Krawczynski, H., Sambruna, R., Kohnle, A., et al. 2001, ApJ, 558, 187

Krimm, H. A., Barthelmy, S. D., Baumgartner, W., et al. 2013, ATel, 4983

Lebrun, F., Leray, J. P., Lavocat, P., et al. 2003, A\&A, 411, L141

Lichti, G. G., Bottacini, E., Ajello, M., et al. 2008, A\&A, 486, 721

Lund, N., Budtz-Jorgensen, G., Westergaard, N. J., et al. 2003, A\&A, 411, L231

Malizia, A., Capalbi, M., Fiore, F., et al. 2000, MNRAS, 312, 123

Mankuzhiyil, N., Ansoldi, S., Persic, \& Tavecchio, F. 2011, ApJ, 733, 14

Maraschi, L., Fossati, G., Tavecchio, F., et al. 1999, ApJ, 526, L81

Mas-Hesse, J. M., Giménez, A., Culhane, L., et al. 2003, A\&A, 411, L261
Massaro, E., Perri, M., Giommi, P., \& Nesci, R. 2004a, A\&A, 413, 489

Massaro, E., Perri, M., Giommi, P., Nesci, R., \& Verrecchia, F. 2004b, A\&A, 422, 103

Massaro, E., Tramacere, A., Perri, M., Giommi, P., \& Tosti, G. 2006, A\&A, 448, 861

Mastichiadis, A., Petropoulou, M., \& Dimitrakoudis, S. 2013, MNRAS, 434, 2684

Mattox, J. R., Bertsch, D. L., Chiang, J., et al. 1996, ApJ, 461, 396

Mukherjee, R., for the VERITAS collaboration, 2013, The innermost regions of relativistic jets and their magnetic fields, Granada (Spain), June 10 14, 2013, http://jets2013.iaa.es/sites/jets2013.iaa.es/files/ imagecache/Mukherjee_Granada_jets2013_rev.pdf

Negoro, H., Suzuki, K., Kawai, N. et al. 2013, ATel, 4978

Nilsson, K., Pasanen, M., Takalo, L. O., et al. 2007, A\&A, 475, 199

Nolan, P. L., Abdo, A. A., Ackermann, M., et al. 2012, ApJS, 199, 31

Padovani, P., \& Giommi, P. 1995, ApJ, 444, 567

Padovani, P., Giommi, P., \& Rau, A. 2012, MNRAS, 422, L48

Paneque, D., D’Ammando, F., Orienti, M., \& Falcone, A. 2013, ATel, 4977

Perlman, E. S., Madejski, G., Georganopoulos, M., et al. 2005, ApJ, 625, 727

Peterson, B. M., Wanders, I., Horne, K., et al. 1998, PASP, 110, 660

Pian, E., Ubertini, P., Bazzano, A., et al. 2011, A\&A, 526, A125

Potter, W. J., \& Cotter, G. 2013, MNRAS, 436, 304

Ravasio, M., Tagliaferri, G., Ghisellini, G., \& Tavecchio, F. 2004, A\&A, 424, 841

Schlafly, E. F., \& Finkbeiner, D. P. 2011, ApJ, 737, 103

Sembay, S., Edelson, R., Markowitz, A., Griffiths, R. G., \& Turner, M. J. L. 2002, ApJ, 574, 634

Semkov, E., Bachev, R., Strigachev, A., et al. 2013, ATel, 4982

Shukla, A., Chitnis, V. R., Vishwanath, P. R., et al. 2012, A\&A, 541, A140

Takahashi, T., Tashiro, M., Madejski, G., et al. 1996, ApJ, 470, L89

Tanihata, C., Urry, C. M., Takahashi, T., et al. 2001, ApJ, 563, 569

Tavecchio, F., Maraschi, L., Pian, E., et al. 2001, ApJ, 554, 725

Tavecchio, F., Ghisellini, G., Bonnoli, G., \& Foschini, L. 2011, MNRAS, 414, 3566

Tosti, G., Fiorucci, M., Luciani, M., et al. 1998, A\&A, 339, 41

Tramacere, A., Giommi, P., Perri, M., Verrecchia, F., \& Tosti, G. 2009, A\&A, 501,879

Ubertini, P., Lebrun, F., Di Cocco, G., et al. 2003, A\&A, 411, L131

Ulrich, M.-H. 1973, Astrophys. Lett., 14, 89

Ulrich, M.-H. 1978, ApJ, 222, L3

Urry, C. M., Scarpa, R., O’Dowd, M., et al. 2000, ApJ, 532, 816

Ushio, M., Tanaka, T., Madejski, G., et al. 2009, ApJ, 699, 1964

Vaughan, S., Edelson, R., Warwick, R. S., \& Uttley, P. 2003, MNRAS, 345, 1271

Westergaard, N. J., Kretschmar, P., Oxborrow, C. A., et al. 2003, A\&A, 411, L257

White, R. J., \& Peterson, B. M. 1994, PASP, 106, 879

Winkler, C., Courvoisier, T. J.-L., Di Cocco, G., et al. 2003, A\&A, 411, L1

Zhang, Y.-H., Cagnoni, I., Treves, A., Celotti, A., \& Maraschi, L. 2004, ApJ, 605,98 